\title{
Biologia e aspectos morfológicos dos estágios imaturos de Automeris naranja Schaus (Lepidoptera, Saturniidae, Hemileucinae)
}

\author{
Alexandre Specht ${ }^{1,2}{ }^{\text {; }}$ Aline C. Formentini ${ }^{1} \&$ Elio Corseuil ${ }^{3}$ \\ ${ }^{1}$ Laboratório de Biologia, Departamento de Ciências Exatas e da Natureza, Campus Universitário da Região dos Vinhedos, \\ Universidade de Caxias do Sul, Caixa Postal 32, 95700-000 Bento Gonçalves, Rio Grande do Sul, Brasil. \\ E-mail: acformen@ucs.br \\ 2 Instituto de Biotecnologia, Universidade de Caxias do Sul. Caixa Postal 1352, 95070-560 Caxias do Sul, Rio Grande do \\ Sul, Brasil. E-mail: aspecht@ucs.br \\ ${ }^{3}$ Livre Docente em Entomologia. Professor Titular aposentado. E-mail: corseuil@via-rs.net
}

\begin{abstract}
Biology and morphological aspects of the immature stages of Automeris naranja Schaus (Lepidoptera, Saturniidae, Hemileucinae). This work aimed to study the biology and the morphology of immature stages of Automeris naranja Schaus, 1898, which is a polyphytophagous hemileucinae and their caterpillars might cause erucism. The biological parameters were obtained under laboratory conditions with temperature: $25 \pm$ $1^{\circ} \mathrm{C}$; UR $70 \pm 10 \%$; photofase of 14 hours, and daily observations. On each developmental phase, morphological and ethologic aspects, as well as duration and viability, were evaluated. In order to link the host plants to the insect it were added data of collected material on field and referred in the bibliography. The life cycle was of approximately 128 days, whose average periods of eggs, caterpillars, pupae and adult phases were 14.89, 69.00, 35.19 and 7.61 days, respectively. The caterpillars passed by six instars with a growth average rate of 1.46. It was observed a high degree of polyphytophagy over 61 host plants belonging in 26 families. The biotic potential was estimated on 1,195,606.994 individuals in the year.
\end{abstract}

KEY WORDS. Biological parameters; caterpillars; erucism; life cycle.

RESUMO. Este estudo objetivou estudar a biologia e aspectos da morfologia dos estágios imaturos de Automeris naranja Schaus, 1898, um hemileucíneo polifitófago cujas lagartas podem provocar erucismo. Os parâmetros biológicos foram obtidos em condições controladas: temperatura, $25 \pm 1^{\circ} \mathrm{C}$; Umidade Relativa, $70 \pm 10 \%$ de UR e fotofase, 14 horas, com observações diárias. Foram avaliados, em cada fase de desenvolvimento, aspectos morfológicos e etológicos, duração e viabilidade. Para relacionar as plantas hospedeiras foram reunidos dados já referidos em bibliografia e de material coletado em campo. O ciclo de vida foi de 128 dias, cujos períodos médios das fases de ovo, lagarta, pupa e adulta foram de 14,89; 69,00; 35,19 e 7,61, respectivamente. As lagartas passaram por seis ínstares e tiveram uma razão média de crescimento de 1,46 . Observou-se um alto grau de polifitofagia sendo relacionadas 61 plantas hospedeiras pertencentes a 26 famílias. $O$ potencial biótico foi estimado em 1195606,994 indivíduos ao ano.

PALAVRAS-CHAVE. Ciclo de vida; erucismo; lagartas; parâmetros biológicos.

O saturniídeo Automeris naranja Schaus, 1898 (Hemileucinae) é amplamente distribuído na região que compreende o Bioma Mata Atlântica e adjacências, do Estado de Pernambuco, no Brasil até o Nordeste da Argentina, incluindo o Paraguai e Uruguai (Lemaire 2002). É uma espécie muito comum, popularmente conhecida como olho-de-pavão-ruivo (BIEZANKo \& BAUCKE 1948, BiezANKo \& Link 1972, Buzzi 1994); apresenta como sinônimos A. aurantiaca Weymer, 1907, A. lineatus Bouvier, 1930 e A. naranja pernambucensis Lemaire, 1973 (LemarRe 2002). Devido à identificação equivocada de MABILDE (1896) esta espécie foi confundida com A. complicata (Walker, 1855), que tem distribuição restrita a Venezuela (Lemaire 2002), sendo referida como tal até a década de 60 (Ronna 1933, 1934, Biezanko \& Freitas 1938, Biezanko \& Seta 1939, Baucke 1960). Na Argentina KöHLER (1935) cita que A. aurantiaca foi descrita por complicata e BiezAnKo et al. (1957) relacionando a fauna do Uruguai referem que todos os trabalhos antigos da região do Rio da Prata (Argentina) que se referem a $A$. naranja utilizam o nome $A$. complicata, sem dúvida baseando-se em Mabilde (1896). Na Argentina esta espécie ainda foi confundida com outra muito semelhante, Automeris umbrosa Weymer, 1906, no trabalho de Schreiter (1943) cujos dados foram compilados por HaYward (1969) e as plantas hospedeiras foram referidas por PASTRANA (2004). Da mesma forma, BouRQuin (1944b, 1948), relaciona o

Revista Brasileira de Zoologia 24 (3): 523-534, setembro 2007 
estudo de $A$. aurantiaca, porém deixa dúvida quanto à identificação citando que pode se tratar de Automeris ater Conte, 1929 que, segundo Lemaire (2002) é um sinônimo de A. umbrosa.

Esta espécie apresenta diversas plantas hospedeiras, especialmente árvores e arbustos (SiLva et al. 1968), sendo referida como praga de extremosa (Ronna 1933, Biezanko \& Freitas 1938) e goiabeira (Ronna 1933). No Rio Grande do Sul A. naranja encontra-se representada em praticamente todas as coleções entomológicas (Corseuil et al. 2002), cujos exemplares foram coletados nas zonas fisiográficas de Missões, Depressão Central, Encosta do Sudeste, Encosta Superior do Nordeste e Encosta Inferior do Nordeste, durante os meses de janeiro (lagarta e adulto), fevereiro, março, abril (lagarta e adulto), maio (lagarta), junho, agosto, setembro, outubro, novembro, dezembro (Specht et al. 2005).

Assim como Automeris illustris (Walker, 1855), A. naranja é facilmente criada em laboratório sendo utilizada em experimentos sobre comportamento na Europa (BASTOK \& BLEST 1958, BLEST 1958, 1959) e criada por amadores (GARDINER 1958, 1982).

Suas lagartas, como nos demais representantes do gênero, são urticantes (Lemaire 2002, Haddad \& Cardoso 2003, Moraes 2003) e por serem comuns são freqüentemente trazidas pelos alunos do Campus Universitário da Região dos Vinhedos, Universidade de Caxias do Sul, recolhidas por vítimas de acidentes.

Apesar de existirem diversos estudos que trazem contribuições sobre a biologia de A. naranja (D'AlmEIDA 1944a, GARDiner 1958, 1982, VilLiard 1969) em nenhum é feito o detalhamento dos principais aspectos biológicos em condições controladas, objetivo do presente estudo.

\section{MATERIAIL E MÉTODOS}

Os dados foram obtidos a partir de uma criação laboratorial mantida em sala climatizada sob temperatura de $25 \pm 1^{\circ} \mathrm{C}$, umidade relativa de $70 \pm 10 \%$ e fotofase de 14 horas, com observações diárias.

Os aspectos morfológicos referentes às fases do desenvolvimento foram avaliados efetuando-se mensurações através de paquímetro digital e microscópio estereoscópico provido de ocular micrométrica com precisão de centésimo de milímetro e balança com precisão de miligrama.

Fase de ovo. As posturas foram recolhidas e acondicionadas em placas de petri, sobre papel filtro umedecido diariamente, até a eclosão das lagartas. Foram conservados vinte ovos para observações morfológicas e mensurações de maior comprimento e largura.

Fase de lagarta. Durante todo o período larval, as lagartas foram alimentadas com folhas novas (ainda em crescimento) de laranjeira-doce (Citrus sinensis (Linn.) Osbeck - Rutaceae) oferecidas em ramos com aproximadamente cinco folhas, renovados diariamente e cujas bases eram introduzidas em frascos de $50 \mathrm{ml}$ com água destilada para manutenção da turgescência. As lagartas foram mantidas um recipiente de vidro de $30 \times 30$ x 45 $\mathrm{cm}$ até a fase de pupa, quando os casulos foram recolhidos. Nes- ta fase as cápsulas cefálicas eram recolhidas diariamente e medidas em sua maior largura. Assim como observado para a maioria dos hemileucíneos (Lemaire 2002) e conforme descrito para $A$. illustris (SРеCHT et al. 2006b), devido ao hábito gregário principalmente nos primeiros ínstares, não foi possível criar as lagartas individualmente para estimar a duração dos mesmos. Desta forma avaliou-se apenas a duração da fase larval individualizando as lagartas quando iniciavam o período de pré-pupa. Para a diferenciação dos ínstares, como referido por PARRA \& HADDAD (1989) organizou-se uma distribuição de freqüências da maior medida das cápsulas cefálica, em intervalos de décimo de milímetros e posterior agrupamento. Ao final das medições, elaborou-se uma curva de distribuição de freqüência visando comparar com o modelo linear de Dyar (1890).

Período de pré-pupa. Este período levou em consideração a data em que a lagarta parou de se alimentar e deixou a planta hospedeira para procurar substrato para a confecção do casulo até a metamorfose em pupa que foi constatada por uma abertura longitudinal nos casulos após o segundo dia da sua elaboração.

Fase de Pupa. Constatada a metamorfose, os casulos contendo as respectivas crisálidas, foram mantidos em recipiente de vidro com capacidade de $500 \mathrm{ml}$ forrado com papel filtro umedecido diariamente, até a emergência dos adultos. Dois dias após a transformação realizou-se a retirada das pupas quando foram efetuadas as medições de maior comprimento, largura e massa, além da identificação do sexo, seguindo-se esquemas de Butt \& Cantu (1962).

Fase adulta. Após a emergência, os adultos foram mantidos aos pares formando 20 casais, em gaiolas cilíndricas de PVC com diâmetro e altura de $20 \mathrm{~cm}$, revestidas com filme plástico na base e tecido tipo "voile" branco na porção superior. Estas gaiolas foram forradas, no fundo e nas laterais com papel filtro e também receberam um graveto. Durante esta fase avaliaramse os períodos de pré, pós e oviposição.

A partir da reunião dos parâmetros biológicos calculouse o potencial biótico, conforme Silveira Neto et al. (1976), considerando-se nula a resistência do ambiente.

Os dados foram analisados através de estatística descritiva com o cálculo das médias e erros-padrões. As médias foram comparadas pelo teste $t$, presumindo variâncias diferentes, ao nível de significância de 95\%.

Exemplares testemunhos da pesquisa foram fotografados e incorporados à coleção do Laboratório de Biologia, Campus Universitário da Região dos Vinhedos, Universidade de Caxias do Sul (CARVI-UCS) e do Museu de Ciências e Tecnologia da PUCRS.

Realizou-se um levantamento das plantas hospedeiras citadas em bibliografia e também foram reunidas informações a partir de lagartas trazidas por estudantes e comunidade em geral ao Laboratório de Biologia do CARVI-UCS, nos últimos quatro anos, cujos exemplares foram mantidos em criação, sempre que possível na própria planta hospedeira. A nomenclatu- 
ra das plantas foi atualizada utilizando-se, principalmente, BACKeS \& NARDino (2001) relacionando-se os nomes vulgares, científicos, família e primeira referência a registrar a planta hóspede; havendo o registro de uma espécie botânica, deixouse de citar as menções apenas ao respectivo gênero pelos demais autores (Tab. I).

Visando obter dados sobre a época de ocorrência dos adultos foram examinados materiais depositados nas coleções sediadas no Rio Grande do Sul: Centro de Pesquisa Agropecuária de Clima Temperado (CAMB), Faculdade de Agronomia, Setor de Entomologia da Universidade Federal do Rio Grande do Sul (FASE); Museu de Ciências da Universidade Católica de Pelotas (MUCP) e Museu Entomológico Ceslau Biezanko (MECB) em Pelotas; Museu Anchieta de Porto Alegre (MAPA), Museu de Ciências e Tecnologia da PUCRS (MCTP), Museu Ramiro Gomes Costa da Fundação Estadual de Pesquisa Agropecuária (MRGC) em Porto Alegre e Coleção do Laboratório de Biologia CARVI-UCS (CUCS), em Bento Gonçalves.

Material examinado. BRASIL, Rio Grande do Sul: Derrubadas, 24.I.2001, R.A. Di Mare leg., 3 machos (MCTP-10405); Barracão, 21.II.2001, R.A. Di Mare leg., 2 machos (MCTP-10407); Lagoa Vermelha, 15.I.1999, A. Specht leg., 1 macho (MCTP11068); Sarandí, 26.I.2001, R. A. Di Mare leg., 4 machos (MCTP10407); Guarani das Missões (antes Guarani), 27.I.1933, C.M. Biezanko leg., 1 fêmea (MECB); Anta Gorda, 24.XII.2005, E. Fronza leg., 1 fêmea (CUCS); 13.XI.2006, E. Fronza leg., 1 macho (CUCS); 03.III.2006, E. Fronza leg., 1 macho (CUCS); 07.V.2006, E. Fronza leg., 1 fêmea (CUCS); 15.XII.2006, E. Fronza leg., 1 fêmea (CUCS); 16.XII.2006, E. Fronza leg., 1 fêmea (CUCS); 16.XII.2006, E. Fronza leg., 1 macho (CUCS); 17.XII.2006, E. Fronza leg., 1 fêmea (CUCS); Monte Belo do Sul, 13.IV.2003, V.L.B. Balestrin leg., 1 fêmea (CUCS); Bento Gonçalves 30.IV.2003, D. Detoni leg., 1 macho (CUCS); 20.VIII.2003, E. Fronza leg., 1 fêmea (CUCS); 09.X.2003, A.Specht leg., 1 macho (CUCS); 21.X.2003, V.L.C. Balestrin leg., 1 fêmea (CUCS); 26.X.2003, M. Gedoz leg., 1 fêmea (CUCS); 11.I.2004, E. Burlani leg., 1 macho (CUCS); 14.I.2004, A.C. Formentini leg., 1 macho (CUCS); 16.I.2004, A.C. Formentini leg., 9 machos (CUCS); 19.I.2004, A.C. Formentini leg., 1 fêmea (CUCS); 19.I.2004, A.C. Formentini leg., 1 macho (CUCS); 20.I.2004, A.C. Formentini leg., 1 fêmea (CUCS); 20.I.2004, A.C. Formentini leg., 1 macho (CUCS); 21.I.2004, A.C. Formentini leg., 2 fêmeas (CUCS); 21.I.2004, A.C. Formentini leg., 1 macho (CUCS); 22.I.2004, A.C. Formentini leg., 1 fêmea (CUCS); 22.I.2004, A.C. Formentini leg., 1 macho (CUCS); 28.I.2004, A.C. Formentini leg., 1 fêmea (CUCS); 18.II.2004, A.C. Formentini leg., 1 fêmea (CUCS); 25.IV.2004, A.C. Formentini leg., 1 macho (CUCS); 16.V.2004, A.C. Formentini leg., 1 fêmea (CUCS); 25.V.2004, A.C. Formentini leg., 1 macho (CUCS); 27.X.2005, A.J. Benedetti leg., 1 macho (CUCS); Caxias do Sul, 04.IV.2003, A.Specht leg., 1 fêmea (CUCS); 05.V.2003, A.Specht leg., 1 fêmea (CUCS); 13.V.2003, A.Specht leg., 1 fêmea (CUCS); Salvador do Sul, 8.V.1994, A. Specht leg., 1 fêmea (MCTP-4042); 03.IX.1996, A. Specht leg., 1 macho (MCTP-
11064); Pareci Novo, X.1947, (sem coletor), 1 fêmea (MAPA); São Leopoldo, X.1940, (sem coletor), 1 macho (MAPA); 17.V.1953, (sem coletor), 1 macho (MAPA); São Sepé, 14.III.1995, Costa \& Link leg., 1 macho (MCTP-8277); Porto Alegre, 01.X.1912, (sem coletor), 1 macho (MAPA); 30.IX.1928, (sem coletor), 16.II.1932, (sem coletor), 1 macho (MAPA); 1 macho (MAPA); 15.II.1938, (sem coletor), 1 macho (MAPA); 16.II.1938, (sem coletor), 1 macho (MAPA); 07.X.1941, (sem coletor), 1 macho (MAPA); 14.II.1945, D.C. Redaelli leg., 1 fêmea (MRGC3438); 09.III.1945, C. Nogueira leg., 1 macho (MRGC-3446); 2.I.1946, (sem coletor), 1 macho (MAPA); 03.I.1946, (sem coletor), 1 fêmea (MAPA); XII.1946, (sem coletor), 1 fêmea, lagarta em ameixa-preta (MAPA); 3.X.1948, (sem coletor), 1 fêmea (MAPA); 25.IX.1948, R.G. Costa leg., 1 macho (MRGC-3447); 10.XII.1953, E. Corseuil leg., 1 macho (MRGC-3441); II.1954, E. Corseuil leg., 1 fêmea (MRGC-3436); XI.1954, E. Corseuil leg., 1 macho, lagarta em pereira (MRGC-3445); 27.XI.1954, E. Corseuil leg., 1 macho (MRGC-3444); 27.XI.1954, E. Corseuil leg., 1 macho (MRGC3452); 04.II.1955, E. Corseuil leg., 1 fêmea (MRGC-3439); 12.I.1955, E. Kober leg., 1 macho (MRGC-3450); 12.I.1955, E. Corseuil leg., 1 macho, lagarta em pereira (MRGC-3451); 22.III.1958, D.C. Redaelli leg., 1 macho (MRGC-3449); 28.X.1959, E. Risi Neto leg., 1 macho, lagarta em maricá (MRGC-03445); 04.IX.1967, (sem coletor), 1 macho (MAPA); 18.X.1966, (sem coletor), 1 macho (MAPA); 24.IX.1970, (sem coletor), 1 fêmea (MAPA); 12.IX.1971, (sem coletor), 1 fêmea (MAPA); 17.I.1974, C. Trois leg., 1 macho, hospedeira Tecoma sp. (FASE - 5804); 09.IX.1974, (sem coletor), 2 fêmeas (MAPA); 09.X.1974, (sem coletor), 2 machos (MAPA); 06.III.1979, Ir. Cláudio leg., 1 fêmea (MAPA); 23.X.1980, R. Beneveti leg., 1 macho (MAPA); 30.I.1995, L.M. Lorini leg., 1 fêmea (MCTP-4307); 12.II.1995, L.M. Lorini leg., 1 fêmea (MCTP-11063); 01.III.1998, R. Rodenbusch leg., 1 macho (MCTP-11062); 20.II.2005, F.P.R. Santos leg., 1 macho (MCTP-14290); Rosário do Sul, 12.I.1972, J. A. Martins leg. Hospedeira Tecoma sp., 1 fêmea (FASE - 5803); Pelotas, 09.X.1938, C.M. Biezanko leg., 1 fêmea (MECB); 12.X.1938, C.M. Biezanko leg., 2 fêmeas (MECB); 13.X.1938, C.M. Biezanko leg., 1 macho (MECB); 16.X.1938, C.M. Biezanko leg., 1 macho (MECB); 19.X.1938, C.M. Biezanko leg., 4 machos (MECB); 15.I.1939, Irmãs Figueiredo leg., 1 macho (MUCP); 17.I.1939, C.M. Biezanko leg., 1 fêmea (MECB); 22.I.1939, C.M. Biezanko leg., 1 fêmea (MECB); 22.I.1939, C.M. Biezanko leg., 1 macho (MECB); 24.I.1939, C.M. Biezanko leg., 1 fêmea (MECB); 31.I.1944, Irmãs Figueiredo leg., 1 macho (MUCP); 10.IX.1946, P. Silva, 1 fêmea (MECB); 21.IX.1946, C.M. Biezanko leg., 1 macho (MECB); 05.I.1947, Irmãs Figueiredo leg., 1 macho (CAMB); 11.I.1947, Irmâs Figueiredo leg., 1 fêmea (CAMB); 04.III.1947, F. Comiel leg., 1 macho (MECB); 07.X.1947, E. Bortach leg., 1 fêmea (MECB); 05.II.1948, R.M. Real leg., 1 fêmea (MECB); 06.X.1949, F. Comiel leg., 1 macho (MECB); 21.I.1950, Irmãs Figueiredo leg., 1 macho (MUCP); 04.I.1951, L. Pamyaqua leg., 1 fêmea (MECB); 10.I.1951, L.F. Coelho leg., 1 fêmea (MECB); 09.VIII.1951, I.A.S. -Instituto Agronômico do Sul), 1 macho (CAMB); 29.III.1954, I. Coelho 
Tabela I. Plantas hospedeiras de Automeris naranja conforme bibliografia e dados obtidos durante o presente estudo.

\begin{tabular}{|c|c|c|c|}
\hline Nome Vulgar & Nome científico & Familia & Referências \\
\hline Acácia-aroma & Acacia dealbata Link & Mimosaceae & BieZANKo et al. (1949) \\
\hline Acácia-comum & Acacia longifolia Willd. & Mimosaceae & BiezANKo \& Seta (1939) \\
\hline Acácia-mimosa & Acacia podalyriifolia A. Cunn. & Mimosaceae & MABILDE (1896) \\
\hline Acácia-mole & Acacia mollissima (Willd.) Lindl. & Mimosaceae & BiezANKo et al. (1974) \\
\hline Acácia-negra & Acacia mearnsi De Willd. & Mimosaceae & Pastrana (2004) \\
\hline Acalifas & Acalypha sp. & Euphorbiaceae & D'Almeida (1944a) \\
\hline Açoita-cavalo & Luehea divaricata Mart. & Tiliaceae & * \\
\hline Ameixeira & Prunus domestica Linn. & Rosaceae & BIEZANKo et al. (1974) \\
\hline Ameixeira-do-Japão & Prunus triflora Roxb. & Rosaceae & Biezanko \& Freitas (1938) \\
\hline Amendoeira-da-praia & Terminalia catappa Linn. & Combretaceae & D'ALMEIDA (1944a) \\
\hline Anoneira & Anona cherimolia Mill. & Annonaceae & BIEZANKo et al. (1974) \\
\hline Araticum-mirim & Rollinia emarginata Schlecht & Annonaceae & * \\
\hline Cafeeiro & Coffea arabica Linnaeus & Rubiaceae & RonNa (1933) \\
\hline Canela-preta & Nectandra megapotamica (Spreng.) Mez & Lauraceae & * \\
\hline Chal-chal & Allophylus edulis (St. Hill.) Radlk. ex Warm. & Sapindaceae & * \\
\hline Carvalho & Quercus sp. & Fagaceae & StONe (1991) \\
\hline Damasqueiro & Prunus armeniaca Linnaeus & Rosaceae & BIEZANKo et al. (1974) \\
\hline Erva-mate & Ilex paraguariensis St. Hil. & Aquifoliaceae & BOURQUIN (1944b) \\
\hline Escumilha & Lagerstroemia flos-reginae Retz. & Lythraceae & BiEZANKo et al. (1949) \\
\hline Espinilho & Acacia caven (Mol.) Mol. & Mimosaceae & Pastrana (2004) \\
\hline Eucalipto-grandis & Eucalyptus grandis W. Hill. (Maiden) & Myrtaceae & * \\
\hline Extremosa & Lagerstroemia indica Linnaeus & Lythraceae & RonNa (1933) \\
\hline Falso-pau-Brasil & Caesalpinea spinosa Linnaeus & Mimosaceae & PAStRana (2004) \\
\hline Giesta & Spartium junceum Linnaeus & Fabaceae & RonNa (1933) \\
\hline Glicínia & Wisteria sinensis (Sims.) Sweet & Fabaceae & Biezanko \& Freitas (1938) \\
\hline Goiabeira & Psidium guajava Linnaeus & Myrtaceae & RonNa (1933) \\
\hline Goiabeira-da-serra & Acca sellowiana (Berg.) Burret & Myrtaceae & BouRQUIN (1944b) *** \\
\hline Guabirobeira & Campomanesia xanthocarpa Berg & Myrtaceae & * \\
\hline Hibisco & Hibiscus rosa-sinensis Linnaeus & Malvaceae & BIEZANKo et al. (1974) \\
\hline lbirá & Phyllostylum rhamnoides (J. Poiss.) Taub. & Ulmaceae & PASTRANA $(2004)^{\star *}$ \\
\hline Ipê & Tecoma sp. & Bignoniaceae & $* * * *$ \\
\hline Ipê-de-jardim & Tecoma stans (Linnaues) Juss. Ex H.B. \& K. & Bignoniaceae & PASTRANA $(2004) * *$ \\
\hline Ipê-roxo & Tabebuia impetiginosa (Mart. Ex DC.) Standley & Bignoniaceae & PAstrana (2004) \\
\hline Jacarandá & Jacaranda mimosaefolia D. Don. & Bignoniaceae & BiEZANKo et al. (1974) \\
\hline Jasmineiro-da-Espanha & Jasminum grandiflorum Linnaeus & Oleaceae & BIEZANKo et al. (1949) \\
\hline Jasmineiro-dos-Açores & Jasminum azoricum Linnaeus & Oleaceae & BiEZANKo et al. (1949) \\
\hline Jasmineiro-indiano & Jasminum angustifolium Ker-Gawl & Oleaceae & Biezanko \& Freitas (1938) \\
\hline Laranjeira-azeda & Citrus aurantium Linnaeus & Rutaceae & RonNa (1933) \\
\hline Laranjeira-doce & Citrus sinensis Osbeck & Rutaceae & Biezanko \& Freitas (1938) \\
\hline Ligustrinho & Ligustrum vulgare Linnaeus & Oleaceae & BIEZANKo et al. (1974) \\
\hline Ligustro & Ligustrum lucidum Aiton & Oleaceae & BIEZANKo et al. (1974) \\
\hline Ligustro-japonês & Ligustrum japonicum Thunb. & Oleaceae & BIEZANKo et al. (1974) \\
\hline Lilás & Syringa vulgaris Linnaeus & Oleaceae & STONE (1991) \\
\hline Limoeiro & Citrus limon (Linnaeus) Burm. & Rutaceae & * \\
\hline
\end{tabular}


Tabela I.Continuação.

\begin{tabular}{|c|c|c|c|}
\hline Nome Vulgar & Nome científico & Familia & Referências \\
\hline Louro-de-saia & Phoebe porphyria Mez. & Lauraceae & Pastrana $(2004) * *$ \\
\hline Macieira-brava & Malus sylvestris Mill. & Rosaceae & BIEZANKo et al. (1974) \\
\hline Madressilva & Lonicera sp. & Caprifoliaceae & BOURQUIN (1944b) *** \\
\hline Mamoneiro & Ricinus communis Linnaeus & Euphorbiaceae & RonNa (1934) \\
\hline Mangueira & Mangifera indica Linnaeus & Anacardiaceae & * \\
\hline Maracujá & Passiflora sp. & Passifloraceae & RONNA (1934) \\
\hline Maricá & Mimosa bimucronata Kuntze & Mimosaceae & BIEZANKo \& Seta (1939) \\
\hline Mimosa-lorentzii & Mimosa lorentzii Griseb & Mimosaceae & PASTRANA (2004) \\
\hline Oliveira & Olea europea Linnaeus & Oleaceae & * \\
\hline Olmo & Ulmus americanus Linnaeus & Ulmaceae & BIEZANKo et al. (1974) \\
\hline Pereira & Pyrus commnunis Linnaeus & Rosaceae & BiezANKo \& Freitas (1938) \\
\hline Pessegueiro & Prunus persica (Linnaeus) Stokes & Rosaceae & BIEZANKo et al. (1949) \\
\hline Pitangueira & Eugenia uniflora Linnaeus & Myrtaceae & * \\
\hline Plátano-ocidental & Platanus occidentalis Linnaeus & Platanaceae & Pastrana (2004) \\
\hline Plátano-oriental & Platanus orientalis Linnaeus & Platanaceae & RonNA (1934) \\
\hline Românzeira & Punica granatum Linnaeus & Lythraceae & * \\
\hline Roseiras & Rosa spp. & Rosaceae & BIEZANKo et al. (1974) \\
\hline Salseiro-chorão & Salix babylonica Linnaeus & Salicaceae & * \\
\hline Salseiro & Salix sp. & Salicaceae & StONe (1991) \\
\hline Taleira-branca & Duranta erecta Linnaeus & Verbenaceae & BIEZANKo et al. (1974) \\
\hline Tipa & Tipuana tipu (Benth.) Kuntze & Cesalpiniaceae & Biezanko \& Freitas (1938) \\
\hline Unha-de-vaca & Bauhinia forficata Link. & Caesalpiniaceae & MABILDE $(1896)$ \\
\hline
\end{tabular}

${ }^{*}$ ) Observações do presente estudo; ${ }^{* *}$ ) plantas referidas por PASTRANA (2004) para A. naranja, entretanto tais plantas foram referidas no trabalho de SCHREITER (1943) para A. umbrosa citando erroneamente A. naranja (LemAIRE 2002); ${ }^{* \star *}$ ) plantas referidas por BouRQUIN (1944b), entretanto a identificação de $A$. naranja é duvidosa; $\left.{ }^{* * \star}\right)$ planta referida em material depositado em coleção.

leg., 1 macho (MECB); 09.X.1956, Austin leg., 1 macho (MECB); 16.II.1963, P. Souza leg., 1 macho (MECB); III.1963, R. Carvalho leg., 1 fêmea (MECB); II.1969, J.C. Cruz leg., 1 macho (MECB); 03.III.1969, Covito leg., 1 macho (MECB); 16.IX.1969, Viana leg., 1 fêmea (MECB); 10.X.1976, Irigoyem leg., 1 macho (MECB); 15.I.1977, C. Silva leg., 1 macho (MECB); 17.I.1977, Niebaker leg., 1 fêmea (MECB); 25.III.1977, Lordan leg., 1 fêmea (MECB); 17.IV.1977, Demarco leg., 1 fêmea (MECB); 18.IV.1979, C.M. Biezanko leg., 1 fêmea (MECB); XI.1996, Guze leg., 1 macho (MECB); X.1999, E. Nassar leg., 1 macho (MECB); VI.1998, A. Dias leg., 1 macho (MECB); XI.1999, W. Kuviate leg., 1 fêmea (MECB).

\section{RESULTADOS E DISCUSSÃO}

\section{Fase de ovo}

Os ovos (Fig. 1) de formato subcilíndrico, achatados no plano longitudinal apresentam maior comprimento (1,695 \pm $0,015 \mathrm{~mm} ; \mathrm{n}=20$ ) entre a micrópila e a extremidade oposta, único ponto onde são fixos ao substrato e a maior largura $(1,545 \pm 0,012 \mathrm{~mm} ; \mathrm{n}=20)$ no primeiro terço abaixo da região micropilar; na porção mais estreita apresentam 0,923 \pm
$0,008 \mathrm{~mm} ; \mathrm{n}=20$. No aspecto geral assemelham-se muito aos ovos descritos para várias espécies do gênero (Lemaire 2002) bem como os já descritos para a mesma espécie (D'Almeida 1944a e, possivelmente, BourQuin 1944b, 1948); os parâmetros morfométricos obtidos assemelham-se aos descritos para a mesma espécie por D'Almeida (1944a) que refere $1,5 \mathrm{~mm}$ no maior comprimento e um pouco menos no menor e Bourquin (1948) que refere $1,75 \mathrm{~mm}$ de comprimento; $1,50 \mathrm{~mm}$ na maior largura e 0,95 $\mathrm{mm}$ de espessura, referente a um representante que pode ser $A$. naranja ou A. umbrosus.

O cório é branco opaco e, logo após a postura, a micrópila é verde-clara, que se torna, nos dias seguintes, enegrecida, com a borda externa esverdeada e próximo à eclosão das lagartas torna-se enegrecida conforme já relatado por D'AlmEIDA (1944a) e Bourquin (1944b, 1948).

O tempo de incubação de aproximadamente 15 dias (Tab. II) se assemelha ao referido por GARDINER (1958) que refere $14 \mathrm{a}$ 21 dias de incubação em temperaturas que variam entre 20 a $25^{\circ} \mathrm{C}$, entretanto D'Almeida (1944a), sem especificar a temperatura, refere um período de aproximadamente 10 dias e BouRQUIN (1948) refere 14 dias a $22^{\circ} \mathrm{C}$. 
Tabela II. Duração média em dias e erros padrões das fases de desenvolvimento de Automeris naranja criada a $25 \pm 1{ }^{\circ} \mathrm{C}$, UR $70 \pm$ $10 \%$ e $14 \mathrm{~h}$ fotofase.

\begin{tabular}{lcr}
\hline \multicolumn{1}{c}{ Fases } & $\mathrm{n}$ & \multicolumn{1}{c}{ Média } \\
\hline Ovo (posturas) & 10 & $14,891 \pm 0,020$ \\
Lagarta & 27 & $65,523 \pm 6,178$ \\
Pré-pupa & 19 & $3,478 \pm 0,451$ \\
Pupa & 18 & $35,191 \pm 8,563$ \\
Adulta (casais) & 20 & $7,608 \pm 0,661$ \\
Pré-oviposição & 16 & $2,861 \pm 0,587$ \\
Oviposição & 16 & $5,712 \pm 1,411$ \\
Pós-oviposição & 16 & $1,433 \pm 0,309$ \\
\hline
\end{tabular}

Apesar dos casais serem mantidos em gaiolas relativamente pequenas, quando comparadas com as utilizadas por GARDINER (1958) que descreve gaiolas de $12 \times 12$ x 18 polegadas, observou-se uma viabilidade média de $94,736 \pm 2,461 \%$ em 19 posturas, corroborando com as observações desse autor que, em condições de laboratório, obteve praticamente 100\% de eclosão.

\section{Fase de lagarta}

As lagartas passaram por seis ínstares (Tab. III), como observado por D'Almeida (1944a) e diferindo de Bourquin (1948). Apesar de GARDINER (1982) referir a ocorrência de um ínstar adicional para lagartas que originaram fêmeas, no presente estudo não foi constatada a ocorrência de ínstares adicionais. As lagartas desta espécie, a exemplo de outros hemileucíneos (Lemaire 2002, Specht et al. 2006), podem apresentar um ínstar adicional, entretanto, devido ao fato da metodologia não ter acompanhado cada lagarta individualmente, não foi possível a sua identificação. BouRQuin (1948), possivelmente referindo-se a mesma espécie, descreve a ocorrência de sete ínstares em uma única fêmea proveniente de um lote que teve diversos problemas durante a criação. A ocorrência destes ínstares adicionais é atribuída ao fato de que o tamanho absoluto das lagartas é o que desencadeia a metamorfose para a fase pupal (Guppy 1969, Ninouth 1975), assim, lagartas que originarão fêmeas devem ter maior tamanho e, portanto podem necessitar maior número de ínstares, principalmente quando não encontram condições ótimas de desenvolvimento.

Observou-se, entre os ínstares, uma razão média de crescimento de 1,464 das cápsulas cefálicas, sendo coerente com o previsto pela regra de DYAR (1890). Resultados recentes com criação de A. llustris (SPECHT et al. 2006b) e Hylesia nigricans (Berg, 1875) (Sреснт et al. 2006a), indicam a ocorrência de valores da taxa média de crescimento muito próximos, em torno de 1,4 a cada ínstar.

No primeiro ínstar (Figs 2-3) logo após a eclosão as lagartas apresentam o corpo com coloração castanho claro, que escurece tornando-se castanho escuro ainda antes de iniciarem a alimentação, o que acontece ainda nas primeiras seis horas. A cápsula cefálica é brilhante, castanho-escura, quase negra com a região clipeal e antenas esbranquiçadas. Os escolos, bastante desenvolvidos apresentam a base castanha com os espinhos mais escuros. Tais características se assemelham muito às descritas por D'Almeida (1944a), Bourquin (1948) e GARdiner (1982). A medida da largura da cápsula cefálica (Tab. III) é muito semelhante à descrita por Bourquin (1948), em torno de $0,85 \mathrm{~mm}$. Como observado nos demais representantes do gênero (Lemaire 2002) e do descrito por Bourquin (1948) e Gardiner (1958) já ao eclodirem suas lagartas apresentam acentuado gregarismo podendo ser vistas andando em fileira e comendo o parênquima das folhas sempre em conjunto (Figs 2-3).

No segundo ínstar, conforme ilustrado nas figuras 4 e 5 (à esquerda) e descrito em D'Almeida (1944a), Bourquin (1948) e GARDINER (1982), a cápsula continua castanho escuro com a região clipeal e antenas esbranquiçadas. No corpo, a área acima dos espiráculos é mais escura, parcialmente esverdeada. São percebidas cinco linhas longitudinais: uma rosada, dorsal menos conspícua; duas amareladas delimitando a fileira de escolos dorsais e uma de igual coloração abaixo dos subdorsais. A linha sub-espiracular só é bem visível e diagonal entre o terceiro e sétimo urômeros; abaixo dela a coloração é castanho claro; pernas torácicas e pseudópodos com coloração castanha mais escura. Escolos mais desenvolvidos, castanho escuro com extremidade amarelada e providos de longas cerdas desta cor que terminam em um longo e fino filamento. A cápsula cefálica (Tab. III) é relativamente maior do que os 1,10 mm observados por BouRquin (1948)

Tabela III. Médias da largura da cápsula cefálica com respectivos erros padrões e razão de crescimento, de lagartas de Automeris naranja alimentadas com folhas de laranjeira (Citrus sinensis), a 25 $\pm 1^{\circ} \mathrm{C}$, UR $70 \pm 10 \%$ e $14 \mathrm{~h}$ fotofase.

\begin{tabular}{|c|c|c|c|}
\hline \multirow{2}{*}{ Ínstar } & \multirow{2}{*}{$\mathrm{N}$} & Largura da cápsula cefálica (mm) & \multirow{2}{*}{$\begin{array}{l}\text { Razão de } \\
\text { crescimento }\end{array}$} \\
\hline & & Média \pm EP & \\
\hline 1 & 94 & $0,863 \pm 0,002$ & - \\
\hline II & 76 & $1,207 \pm 0,006$ & 1,398 \\
\hline III & 72 & $2,124 \pm 0,012$ & 1,760 \\
\hline IV & 71 & $2,812 \pm 0,026$ & 1,324 \\
\hline V & 68 & $4,033 \pm 0,032$ & 1,434 \\
\hline VI & 27 & $5,665 \pm 0,078$ & 1,405 \\
\hline
\end{tabular}

As lagartas de terceiro ínstar, conforme ilustrado nas figuras 5 (espécimes mais escuros) e 6 (exemplar da esquerda) e concordando com as observações de D'Almeida (1944a), BOURQUin (1948) e GARDINER (1982) observa-se que a coloração geral de todo corpo torna-se enegrecida enquanto que a cápsula cefálica torna-se castanho, mais clara que no ínstar anterior. A linha médio dorsal torna-se inconspícua enquanto que as duas que delimitam a fileira de escolos dorsais tornam-se ocre- 

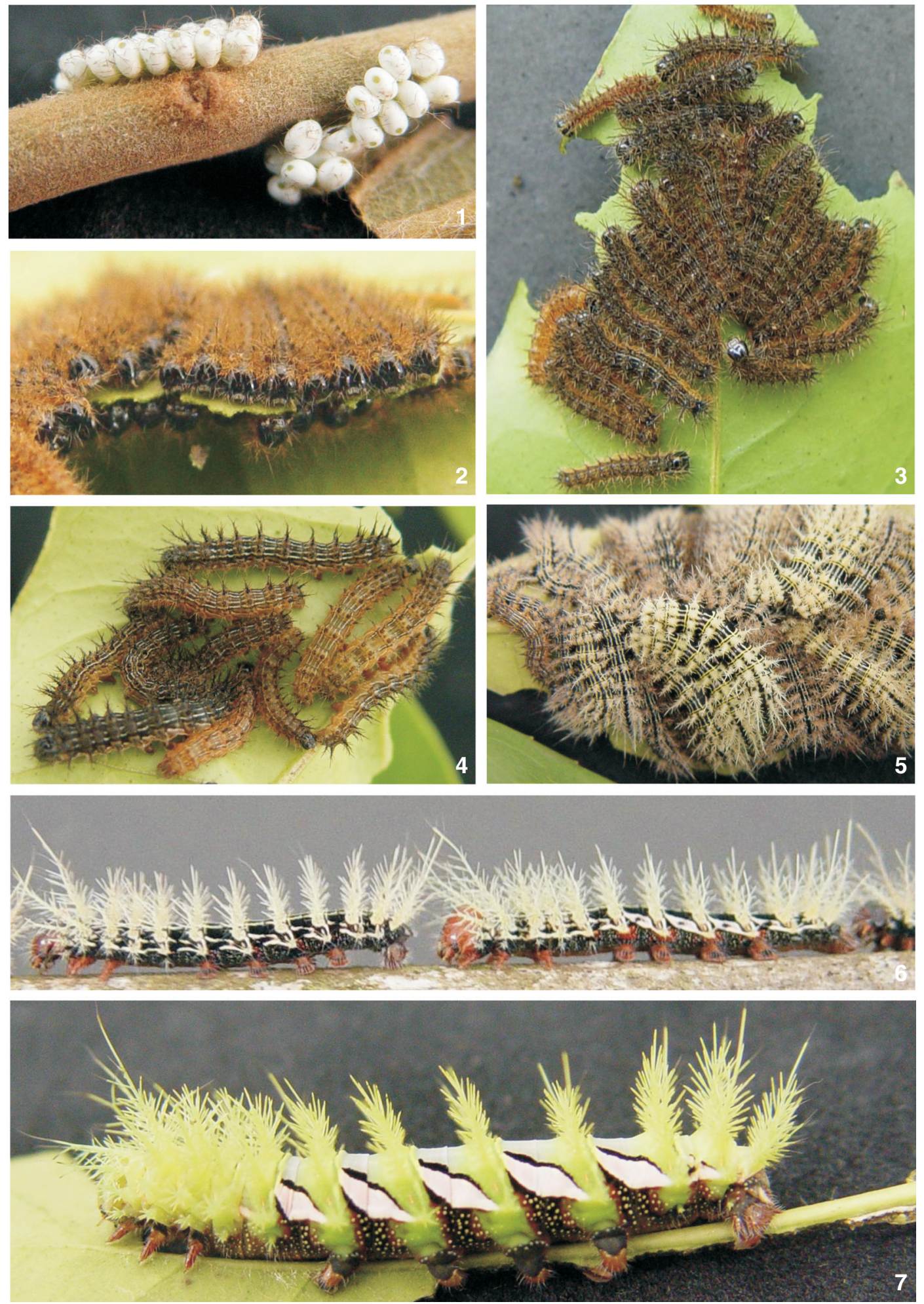

Figuras 1-6. Automeris naranja: (1) ovos; (2-3) lagartas de primeiro ínstar; (4) lagartas de segundo ínstar; (5) vista dorsal das lagartas de terceiro e quarto ínstar; (6) vista lateral das lagartas de terceiro (esquerda) e quarto ínstar (direita); (7) lagarta de quinto ínstar. 
amarelado, interrompidas em cada segmento por uma linha transversal de coloração idêntica imediatamente após os escolos. A linha abaixo dos escolos subdorsais torna-se levemente rosada e tende a se unir à subespiracular, que têm a mesma coloração, após cada espiráculo, especialmente entre o terceiro e o sétimo urômeros. Abaixo da linha subespiracular a coloração de fundo é negra com muitos pontos esbranquiçados, pernas torácicas e pseudópodes castanhos. Escolos mais desenvolvidos que no ínstar anterior, de coloração ocre-rosada (Fig. 5 exemplares mais rosados), providos de longas cerdas desta cor que terminam em um longo e fino filamento. A cápsula cefálica (Tab. III) é bastante maior do que os 1,60 mm observados por BOURQUIN (1948)

No quarto ínstar, conforme descrito em D’Almeida (1944a) Bourquin (1948) e Gardiner (1982), as lagartas mantêm a coloração de fundo negra, porém os escolos tornam-se maiores e de coloração amarelo esverdeado, muito vivo conforme destacado em alguns exemplares da figura 5; as duas linhas que delimitam a fileira de escolos dorsais tornam-se mais evidentes com coloração igual a dos escolos, interrompidas em cada segmento pela linha transversal já descrita no ínstar anterior. A linha abaixo dos escolos subdorsais torna-se rosada e se une à subespiracular, de mesma coloração, formando bandas, especialmente entre o terceiro e o sétimo urômeros, na maioria dos exemplares (Fig. 6 - exemplar da direita). Abaixo da linha subespiracular como descrito para o ínstar anterior. Cápsula cefálica castanho-avermelhada. Entretanto, neste ínstar, não foi observado o vértice negro nos escolos do protórax e mesotórax descritos por Bourquin (1948). A cápsula cefálica (Tab. III) continua sendo muito maior que os 2,45 $\mathrm{mm}$ observados por BouRQuin (1948).

No quinto instar (Fig. 7) a coloração de fundo confere com a descrita por D'Almeida (1944a) e GARDINER (1982), porém difere da descrita por Bourquin (1948) que ainda é muito semelhante a do ínstar anterior. É verde claro amarelado sobre os segmentos torácicos e abdominais. Notam-se em cada lado, do segundo ao oitavo urômeros, especialmente desenvolvidas entre o terceiro e sétimo, largas faixas oblíquas rosadas, bordejadas de negro. Estas faixas começam nos limites dos flancos com o ventre e terminam na região subdorsal do urômero seguinte, nesta posição parte a linha transversal muito próxima e de coloração igual à dos escolos, já verificada nos dois ínstares anteriores, agora dividida por uma fina linha negra. A primeira faixa rosada lateral parte da porção subespiracular distal do terceiro urômero finalizando no segundo; a última inicia no oitavo e estende-se até a região subdorsal do sétimo urômero. O oitavo urômero apresenta uma listra semelhante, porém muito estreita e longitudinal no limite com a região ventral do sétimo, com um filete transversal negro profundo. Espiráculos amarelo-ocre. Cabeça verde-clara tendendo ao ocre. Área subespiracular castanha com regiões inter-segmentares enegrecidas salpicadas de branco e a região abaixo acima dos pseudópodes verde igual ao dos escolos. Pernas torácicas casta- nho claro e inserção negra; pseudópodos negros com porção distal castanha. Ventre pardo claro passando lateralmente para o pardo avermelhado, onde se observa uma coloração negra esmaecida. A cápsula cefálica (Tab. III) é bem maior do que os 3,40 mm observados por BouRQuin (1948)

No sexto ínstar a coloração de fundo, semelhante ao descrito por Mabilde (1896), D'Almeida (1944a), Bourquin (1948), GARDINER (1982) e LEMAIRE (2002) é verde-claro amarelado sobre os segmentos torácicos e abdominais (primeiro, parte anterior do segundo, parte posterior do sétimo, oitavo e nono, também no meio do terceiro, quarto quinto e sexto); cinza-azulado ou cinza-pérola cobre a porção posterior do tergo do segundo urômero, estendendo-se até o sétimo (somente na porção anterior deste). Em cada segmento a cor cinza pérola encontra-se separada da cor do fundo, logo após os escolos, na sua porção posterior, por um filete transversal verde amarelado, precedido de outro, paralelo e verde escuro que corresponde a linha transversal dos ínstares anteriores. As faixas oblíquas tornam-se brancas, ligeiramente róseas, cuja borda negra torna-se mais estreita que no ínstar anterior. Espiráculos como no ínstar anterior. Cabeça da cor do corpo. Nono segmento abdominal com a extremidade matizada de esverdeado e róseo. Pernas torácicas castanhas com a extremidade avermelhada. Placas anais da mesma cor da placa do nono segmento abdominal tendo nos bordos pêlos brancos. Pseudópodes castanhos, com uma mancha escura na região distal não se estendendo até a extremidade distal, com muitos pêlos brancos. Ventre pardo claro passando lateralmente para o pardo avermelhado, onde se observa uma coloração negra esmaecida. Toda esta região apresenta muitas manchas pequenas branco-amarelado, sobre as quais está implantado um curto pêlo claro. Todos os escolos têm coloração verde igual a do corpo, com toda a haste eriçada de duros espinhos de idêntica cor. Assim como descrito por D'Almeida (1944a) a maioria das lagartas apresenta os escolos amarelo vivo próximo ao esverdeado, enquanto que algumas os apresentam cinza-azulado muito claro. A cápsula cefálica (Tab. III) é maior do que os 4,20 e 5,30 mm observados por Bourquin (1948) no exemplar de sexto e sétimo ínstar.

Durante cada ínstar foram observadas diferenças bastante significativas na coloração das lagartas o que justifica pequenas variações encontradas entre a descrição deste estudo e dos trabalhos de D'Almeida (1944a) e Bourquin (1948).

As lagartas de cada postura mantiveram-se em agregações desde a eclosão até o final do quarto ínstar, após formaram grupos menores de até 10 indivíduos ou dispersaram-se individualmente; no último instar não foi observado nenhum gregarismo. Depois de completado o desenvolvimento, as lagartas cessaram a alimentação, procuraram esconderijos, especialmente entre folhas que foram unidas para a confecção de um casulo (Fig. 9) bastante resistente e impermeável construído com seda de coloração âmbar.

A duração total da fase de lagarta (Tab. II) de pouco mais de dois meses é semelhante à descrita por D'Almeida (1944a) 

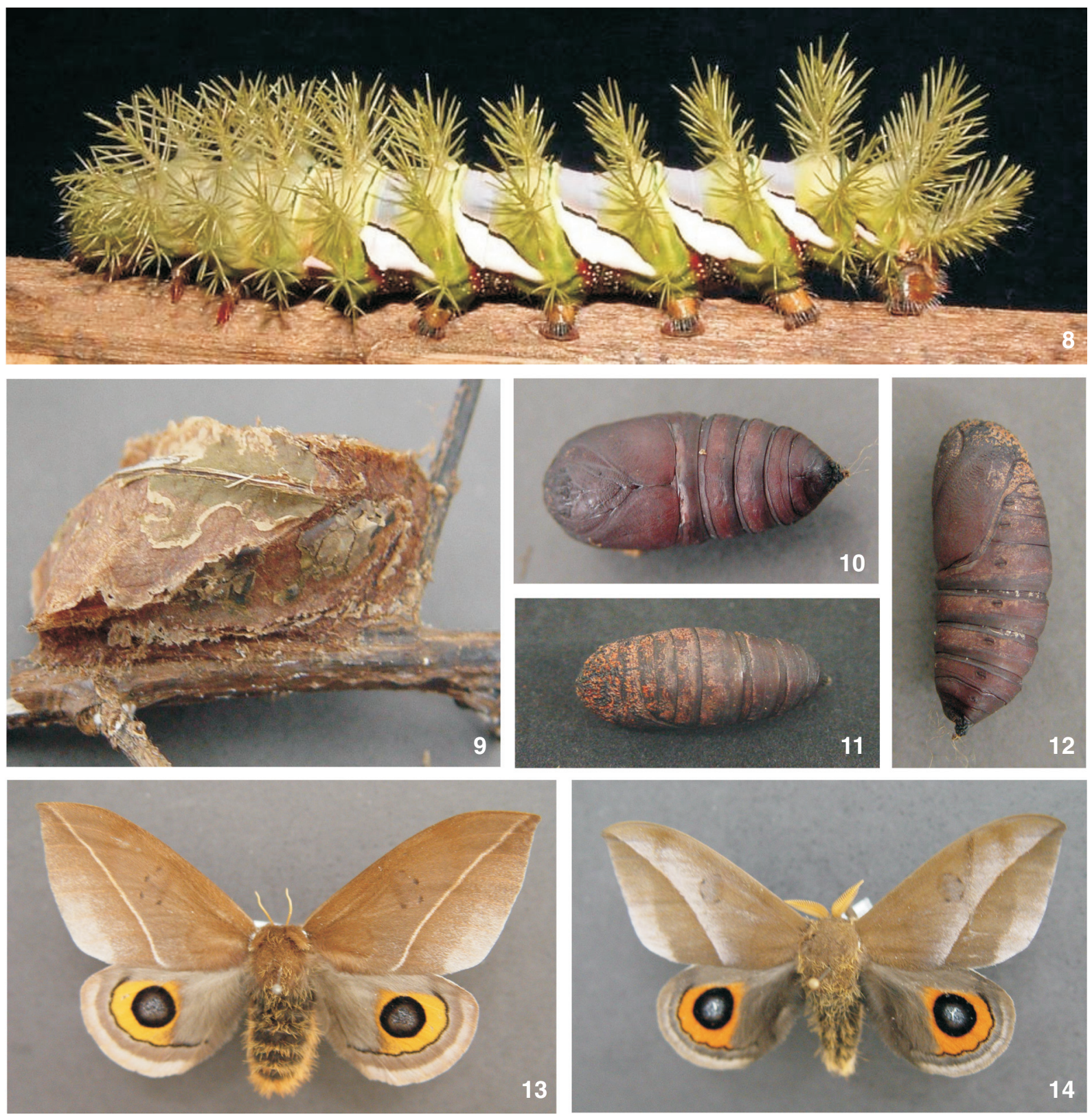

Figuras 8-16. Automeris naranja: (8) lagartas de sexto; (9) casulo cujas folhas externas foram removidas; (10) pupa em vista ventral; (11) pupa em vista dorsal; (12) pupa em vista lateral; (13) fêmea adulta; (14) macho adulto.

para a mesma espécie e de BouRquin (1948). De três agregações com 158, 186 e 234 lagartas foram obtidas 553 pupas representando 95,6\% de viabilidade da fase.

Desconsiderando-se as citações duvidosas, conforme detalhes expressos na tabela I foram relacionadas 61 plantas hóspedes pertencentes a 26 famílias, destacando-se as representantes de Mimosaceae, Oleaceae e Rosaceae. O registro de grande variedade de hospedeiros indica facilidade de adaptação a dife- rentes grupos vegetais relacionando-se esta espécie como polifitófaga, concordando com as observações de D'Almeida 1944b e GARDINER 1958. Diversas espécies deste gênero são consideradas polifitófagas Lemaire (2002), em nosso meio a espécie de Automeris com maior número de plantas hospedeiras registradas é Automeris illustris (Walker, 1855) com 51 plantas pertencentes a 28 famílias (Sреснт et al. 2006b), entretanto Automeris io (Fabricius, 1775) apresenta diversidade de plantas

Revista Brasileira de Zoologia 24 (3): 523-534, setembro 2007 
hospedeiras muito maior, havendo indicação de 120 espécies incluídas em 34 famílias (STONe 1991).

Observou-se que, durante o desenvolvimento, as lagartas desta espécie ao contrário do observado em A. illustris (Sреснт et al. 2006b), apresentam certa facilidade em se alimentar de diferentes plantas hospedeiras, principalmente nos dois ínstares finais. Assim, 31 exemplares trazidos ou enviados por leigos ao laboratório, sem a indicação da planta hospedeira, foram alimentados com sucesso utilizando laranjeira (5), pereira (8), goiabeira (9) e extremosa (9). GARDINER (1958) também relata a facilidade de mudar a planta hospedeira especialmente nos últimos ínstares. Assim, esta espécie pode ser enquadrada na categoria das espécies generalistas que se alimentam de 5 a 10 plantas hospedeiras diferentes, deslocando-se de planta para outra fugindo do ataque de predadores, não retornando para a planta original, normalmente nos dois últimos ínstares (JANSEN 2003). A espécie criada por BouRQuin (1948) também foi alimentada com madressilva até o final do segundo ínstar e depois com goiabeira-da-serra.

No período de pré-pupa, como já relatado para os demais representantes do gênero, observou-se que as lagartas tornaramse mais escuras, e reduziram de tamanho (Lemaire 2002). Utilizaram mais da metade do período desta fase (Tab. II) para confeccionar os casulos rústicos de parede única, parcialmente resistentes e impermeáveis utilizando fios grossos de seda de cor âmbar, unindo folhas ou outros restos vegetais como descrito para A. illustris (SPECHT et al. 2006b). Das 553 lagartas, que formaram casulos, 538 transformaram-se em pupas correspondendo a uma viabilidade de $97,288 \%$.

\section{Fase de pupa}

As fêmeas $(n=20)$ foram significativamente maiores que os machos ( $\mathrm{n}=20)$, apresentando massa de 1,882 $\pm 0,056 \mathrm{~g}$ e $1,393 \pm 0,041$; maior comprimento de 26,185 $\pm 0,305 \mathrm{~mm}$ e $23,889 \pm 0,299 \mathrm{~mm}$ e maior largura lateral 11,095 $\pm 0,167 \mathrm{~mm} \mathrm{e}$ $10,010 \pm 0,135 \mathrm{~mm}$, respectivamente. O valor do maior comprimento se assemelha bastante dos $25 \mathrm{~mm}$ descritos por D'AlmeidA (1944a) e 26,20 mm de comprimento e 11,60 mm de largura referidos para a espécie estudada por BouRQUin (1944b, 1948).

A morfologia da pupa condiz com as descrições de D'ALMEIDA (1944a) e Bourquin (1948) que referem coloração geral (Figs 1012) castanho escuro, com a região dorsal, especialmente até o quarto urômero com matizes avermelhadas (Figs 11 e 12). Face dorsal dos primeiros urômeros apresenta finas carenas transversais, sendo que os posteriores são destituídos sem impressões visíveis, exceto na porção anterior, junto da inserção onde observam-se finas saliências longitudinais. Pterotecas, ceratotecas e podotecas muito rugosas, sendo que as carenas das pterotecas dirigem-se para todas as direções. Pterotecas terminam perpendicularmente ao eixo do corpo aproximadamente na metade do quarto urômero. Tórax com ornado de grânulos grosseiros muito salientes. Cremáster muito rugoso e com ganchos.

De 272 pupas femininas e 266 masculinas emergiram 512 adultos correspondendo a uma taxa de 95,1\% de sobrevivência.
A razão sexual de 0,506 não difere da razão que prevê igualdade entre a ocorrência de indivíduos de cada sexo. Não houve diferença significativa entre sexos para a duração com um mínimo de 31 e um máximo de 48 dias. Nas condições em que foi conduzida a criação laboratorial não foi verificada a diapausa pupal, referida por autores como Mabilde (1896), D'Almeida (1944a) e GARDINER (1958) e que também é observada em outras espécies do gênero (Lemaire 2002). D’Almeida (1944a) refere que esta espécie normalmente é bivoltina, podendo ocorrer uma geração adicional, enquanto GARDINER (1858) refere, para as condições climáticas da Europa, duas gerações anuais, sendo que uma apresenta pupas diapausantes para sobreviver ao inverno.

As comparações das descrições sobre ovo, lagarta e pupa feitas por BouRQuin (1948) com as observações do presente estudo indicam que, caso aquele autor tenha criado $A$. umbrosa, as fase imaturas de ambas espécies são muito semelhantes. LEMAIRE (2002) destaca que não há diferenças conspícuas entre as lagartas de último ínstar destas espécies.

Apesar de BiEzAnKo (1986) referir que suas pupas são parasitadas por Enicospilus americanus Christ 1791 (Hymenoptera, Ichneumonidae), não foi observada a emergência de parasitóides em laboratório.

\section{Fase adulta}

A emergência dos adultos foi observada entre as 16 e 22 horas, semelhante ao descrito por D'Almeida (1944a). Tanto os insetos adultos provenientes da criação quanto os coletados a campo, descritos no material estudado, apresentaram fêmeas (Fig. 13) significativamente maiores $(p<0,05)$ que os machos (Fig. 14). A envergadura das fêmeas e machos oriundos da criação foi de $69,873 \pm 2,566 \mathrm{~mm}(\mathrm{n}=20)$ e $64,346 \pm 2,175 \mathrm{~mm}(\mathrm{n}=20)$ enquanto que os coletados em campo foi de $65,645 \pm 3,867 \mathrm{~mm}$ $(\mathrm{n}=32)$ e $62,431 \pm 3,104 \mathrm{~mm}(\mathrm{n}=43)$, respectivamente. O tamanho dos exemplares criados em laboratório foi um pouco superior aos coletados em campo, indicando que a planta utilizada como alimento supriu bem as necessidades da espécie, que, como a maioria dos hemileucíneos, se alimenta apenas na fase larval (Lemaire 2002). Tanto a envergadura média observada em exemplares de laboratório quanto dos coletados em campo é condizente com a observação de Lemaire (2002) que indica uma variação de $58-79 \mathrm{~mm}$ para fêmeas e $58-79 \mathrm{~mm}$ para machos.

Não houve diferenças significativas entre longevidade dos adultos (Tab. II) que é muito curta e, presumivelmente encontra-se relacionada com o fato dos hemileucíneos não se alimentarem durante a fase adulta (Lemaire 2002). Esta espécie, apresentou período de oviposição mais prolongado que a préoviposição, conforme observado para H. nigricans (SРеCHT et al. 2006a) e A. illustris (SресHT et al. 2006b).

Observou-se que cada fêmea deposita em média 318,245 $\pm 36,897$ ovos $(\mathrm{n}=40)$. Entretanto este número é bastante superior aos 150-200 ovos relatados por GARDINER (1958). As fêmeas fixam os ovos ao substrato, geralmente em um ramo ou folha da planta hospedeira, em duas fileiras ou em pequenos grupos, por vezes, uns sobre os outros, conforme descrito por GARDINER (1958). 
O ciclo de vida completo, desconsiderando o período de pós-oviposição (Tab. II) foi de 127,6 dias, o que corresponde a 2,8 gerações anuais. Estes resultados sugerem que dependendo da latitude, esta espécie pode apresentar número variável de gerações, como observado para $A$. io, que pode apresentar de uma geração ao norte até três ou quatro na Flórida e sudeste do Texas (Lemaire 2002).

Considerando-se a sobrevivência da fase de ovo até adulto, a viabilidade foi de $83,9 \%$. Desta forma cada fêmea é capaz de gerar em média 267,06 descendentes. Assim, nas condições do presente estudo, a estimativa do potencial biótico desta espécie foi de 1195606,944 indivíduos.

Do material examinado nas coleções apenas deixaram de ser localizados adultos coletados no mês de julho no Rio Grande do Sul. Assim como observado para A. illustris (Sреснт et al. 2006a) foram constatados dois picos; um no início da primavera, principalmente em outubro e outro no verão, principalmente entre março e abril. Tais dados sugerem que neste Estado, em função das condições ambientais, esta espécie seja bivoltina como A. io, cujo período de desenvolvimento prolonga-se nos meses de inverno, provavelmente com a passagem por uma diapausa de inverno (Lemaire 2002) na fase de pupa como referido para A. coresus na Argentina (Bourquin 1944b), embora a mesma não tenha sido observada em nossas condições laboratoriais.

\section{AGRADECIMENTOS}

Aos curadores Eduardo J.E. Silva (MECB), Élvia E.S. Vianna (MUCP), Fernando R. Meyer (MAPA), Mirtes Melo (CAMB) e Vera R.S. Wollf (MRGC) pelo acesso e auxílio prestado durante o exame dos materiais. À Liane T. Dorneles pela fotografia correspondente à figura 8. À FAPERGS, pela concessão da Bolsa de Iniciação Científica (Processo 02/508357) à segunda autora e pelo Auxílio Financeiro (Processo 02/1739.6).

\section{REFERÊNCIAS BIBLIOGRÁFICAS}

BACKeS, A. \& M. NARDino. 2001. Nomes populares e científicos de plantas do Rio Grande do Sul. São Leopoldo, UNISINOS, Coleções Fisionomia Gaúcha, $2^{a}$ ed., 202p.

BASTOCK, M. \& A.D. Blest. 1958. An analysis of behaviour sequences in Automeris aurantiaca Weym. (Lepidoptera). Behaviour 12 (3): 234-284.

BAUCKE, O. 1960. Notas entomológicas III. O gênero Automeris Hübner, 1819 (Lepidoptera, Hemileucidae) no Rio Grande do Sul. Iheringia, Série Zoologia 12: 11-19.

BLEST, A.D. 1958. Interaction between consecutive responses in a hemileucid moth, and the evolution of insect communication. Nature 181 (4615): 1077-1078.

BlEst, A.D. 1959. Central control of interactions between behaviors patterns in a hemileucine moth. Nature 184 (4693): 1164-1165.

Biezanko, C.M. 1986. Adelocephalidae, Saturniidae, Lasiocam- pidae, Eupteritidae e Lymantriidae da Região Missioneira do Rio Grande do Sul. Revista do Centro de Ciências Rurais 16 (2): 89-112.

BiezAnKo, C.M. \& D. Link. 1972. Nomes populares dos lepidópteros do Rio Grande do Sul. Boletim Técnico de Defesa Fitossanitária da Universidade Federal de Santa Maria 4: $1-15$.

Biezanko, C.M. \& F.D. Seta. 1939. Catálogo dos insetos encontrados em Rio Grande e seus arredores. Pelotas, Universal, $15 \mathrm{p}$.

Biezanko, C.M. \& O. Baucke. 1948. Nomes populares dos lepidópteros do Rio Grande do Sul. Agros 1 (4): 164-177.

Biezanko, C.M. \& R.G. Freitas. 1938. Catálogo dos insetos encontrados na cidade de Pelotas e seus arredores. Fasc. 1 Lepidópteros. Boletim da Escola de Agronomia "Eliseu Maciel" 25: 1-32.

Biezanko, C.M.; A. Ruffinelli \& C.S. Carbonell. 1957. Lepidoptera Del Uruguay -Lista anotada de espécies. Revista de la Faculdad de Agronomía 46: 1-152.

Biezanko, C.M.; A. Ruffinelui; D. Link. 1974. Plantas y otras sustâncias alimentícias de las orugas de los lepidópteros uruguayos. Revista do Centro de Ciências Rurais 4 (2): 107148.

Biezanko, C.M.; R.E. Bertholdi \& O. Baucke. 1949. Relação dos principais insetos prejudiciais observados nos arredores de Pelotas nas plantas cultivadas e selvagens. Agros 2 (3): 156213.

Bourquin, F. 1944a. IX. Observaciones sobre Automeris coresus (Boisduval), 1859 -Lep. Fam. Hemileucidae, p. 33-35. In: F. BoURQUin (Ed.). Mariposas Argentinas - vida, desarrollo, costumbres y hechos curiosos de algunos lepidópteros argentinos. Buenos Aires, Ferrari Hermanos Bartolome Mitre, 223p.

Bourquin, F. 1944b. XLVI - Observaciones sobre Automeris aurantiaca Weymer, 1907. (Lep. Fam. Hemileucinae), p. 177179. In: F. Bourquin. Mariposas Argentinas - vida, desarrolo, costumbres $\mathrm{Y}$ hechos curiosos de algunos lepidópteros argentinos. Buenos Aires, Ferrari Hermanos Bartolome Mitre, 213p.

Bourquin, F. 1948. Metamorfosis de Automeris naranja Schaus, 1898 (aurantiaca Weym.) (Lep. Het. Hemileucidae). Acta Zoologica Lilloana 5: 69-80.

ButT, B.A. \& E. CANTU. 1962. Sex determination of lepidopterous pupae. Washington, USDA, $7 \mathrm{p}$.

Buzzi, Z.J. 1994. Coletânea de nomes populares de insetos do Brasil. Curitiba, UFPR, 230p.

Corseuil, E.; A. Specht \& C. Lang. 2002. Saturniídeos (Lepidoptera, Saturniidae) registrados para o Rio Grande do Sul, Brasil. I. Hemileucinae. Biociências 10 (2): 147-155.

D'Almeida, R. F. 1944a. Estudos biológicos sobre Lepidopteros Automeris aurantiaca Weymer, 1907. Arquivos de Zoologia 4: 33-72.

D'Almeida, R.F. 1944b. Algumas notas sobre a fauna de lepidóp- 
teros de Monte Alegre. Papéis Avulsos do Departamento de Zoologia 6 (3):21-28.

Dyar, H.G. 1890. The number of molts of lepidopterous larvae. Psyche 5: 420-422.

GardineR, B.O.C. 1958. Rearing Automeris aurantiaca Weymer. Bulletin of the Amauter Entomologistis' Society 17: 1415.

GARDINER, B.O.C. 1982. A silkmoth rearer's handbook. Hanworth, The Amateur Entomologist Society, $3^{\text {rd }}$ ed., 255p.

Guppy, J.C. 1969. Some efects of temperature on the immature stages of the armyworm, Pseudaletia unipuncta (Lepidoptera: Noctuidae), under controlled condictions. The Canadian Entomologist 101: 1320-1327.

Haddad, V. \& J.L.C. Cardoso. 2003. 22 - Erucismo e Lepidopterismo, p. 220-223. In: J.L.C. Cardoso; F.O.S. FranÇA; F.H. Wen; C.M.S. Málaque \& V. Haddad (Eds). Animais peçonhentos no Brasil - biologia, clínica e terapêutica dos acidentes. São Paulo, Sarvier, 468p.

Hayward, K.J. 1969. Datos para el studio de la ontogenia de lepidópteros argentinos. Miscelánea del Instituto Miguel Lillo 31: 1-142.

JANZEN, D.H. 2003. How polyphagous are Costa Rican dry forest saturniid moth caterpillars?, p. 369-379. In: Y. BASSET; R. Kitching; S. Miller \& V. Novotny (Eds). Arthropods of Tropical Forests: Spatio-Temporal Dynamics and Resource Use in the Canopy. Cambrigde, Cambridge University Press, 490p.

KöHLER, P. 1935. Notas sobre Automerinae (Lep. Saturn.) argentinos. Revista de la Sociedad Entomologica Argentina 7: 79-91.

Lemaire, C. 2002. The Saturniidae of America - Hemileucinae. Keltern, Goecke \& Evers, 1388p.

Mabilde, A.P. 1896. Guia práctico para os principiantes collecionadores de insectos, contendo a descrição fiel de perto de 1000 borboletas com 180 figuras lytographadas em tamanho, formas e desenhos conforme o natural. Estudo sobre a vida de insectos do Rio Grande do Sul e sobre a caça, classificação e conservação de uma coleção mais ou menos regular. Porto Alegre, Gunlach \& Schuldt, 238p.

Moraes, R.H.P. 2003. 21. Lepidópteros de importância médica, p. 211-219. In: J.L.C. Cardoso; F.O.S. França; F.H. Wen; C.M.S. Málaque \& V. Haddad (Eds). Animais peçonhentos no Brasil - biologia, clínica e terapêutica dos acidentes. São Paulo, Sarvier, 468p.

Ninouth, H.F. 1975. A threshold size for metamorphosis in the tobacca hornworm, Manduca sexta (L.). The Biological Bulletin 149: 214-225.

Parra, J.R.P. \& M.L. Haddad. 1989. Determinação do número de ínstares de insetos. Piracicaba, FEALQ, 49p.

PASTRANA, J.A. 2004. Los lepidópteros argentinos: sus plantas hospedadoras y otros sustratos alimenticios. Buenos Aires, Sociedad Entomológica Argentina, 350p.

RonNa, E. 1933. Catálogo dos insetos até hoje encontrados nas plantas do Rio Grande do Sul. Egatéa 18: 47-53, 96-100, 197-202, 275-278.

Ronna, E. 1934. Catálogo dos insetos até hoje encontrados nas plantas do Rio Grande do Sul. Egatéa 19: 15-20, 115-120, 277-278.

Schreiter, R. 1943. Notas entomo-biológicas y otras. Acta Zoológica Lilloana 1943: 7-44.

Silva, A.G.A.; C.R. Gonçalves; D.M. Galvão; A.J.L. Gonçalves; J. GoMES; M.N. SiLVA \& L. SIMONI. 1968. Quarto catálogo dos insetos que vivem nas plantas do Brasil: seus parasitos e predadores. Parte II, $1^{\circ}$ tomo, Insetos, hospedeiros e inimigos naturais. Rio de Janeiro, Ministério da Agricultura, 622p.

Silveira Neto, S.; O. Nakano; D. Barbin \& N.A. Villa Nova. 1976. Manual de ecologia dos insetos. São Paulo, Ceres, 419p.

Specht, A.; E. Corseuil. \& A.C. Formentini. 2005. Lepidópteros de importância médica ocorrentes no Rio Grande do Sul. III. Saturniidae, Hemileucinae. Biociências 13 (2): 149-162.

Specht, A.; A.C. Formentini \& E. Corseuil. 2006a. Biologia de Hylesia nigricans (Berg) (Lepidoptera, Saturniidae, Hemileucinae). Revista Brasileira de Zoologia 23 (1): 248-255.

Specht, A.; A.C. Formentini \& E. Corseuil. 2006b. Biologia de Automeris illustris (Walker) (Lepidoptera, Saturniidae, Hemileucinae). Revista Brasileira de Zoologia 23 (2): 537-546.

STONE, S.E. 1991. Foodplants of World Saturniidae. Memoirs of the Lepidopteroists' Society 4: 1-186.

VILLIARD, P. 1969. Moths and how to rear then. New York, Funk and Wagnalls, 242p.

Recebido em 18.I.2007; aceito em 24.VI.2007. 